Monatsschr Kinderheilkd 2012 • 160:489-490 DOI 10.1007/s00112-011-2621-1

Online publiziert: 29. März 2012

(c) Springer-Verlag 2012

\title{
M. Heckmann
}

Abteilung für Neonatologie und Pädiatrische Intensivmedizin,

Zentrum für Kinder- und Jugendmedizin, Universitätsmedizin Greifswald

\section{Neu- und Frühgeborenen- ernährung konkret}

Discharge-Formulanahrungen kommerziell zur Verfügung.

„To avoid underfeeding and overfeeding" bei der Ernährung von Frühgeborenen nach der Entlassung entwickelte sich zu einer anspruchsvollen Aufgabe des Pädiaters in der Praxis in Zusammenarbeit mit den Nachsorgeambulanzen der Perinatalzentren.

Das zweite Konsensuspapier betrifft ein zwischen den Berufsgruppen im praktischen klinischen Alltag immer wieder diskutiertes Thema: das Zufüttern bei gestillten reifen Neugeborenen und späten Frühgeborenen. Wie in der Veröffentlichung vorangestellt, besteht in der Fachwelt Einigkeit darüber, dass ausschließliches Stillen für rund 6 Monate die optimale Ernährung für das Neugeborene und den Säugling ist. Das Konsensuspapier orientiert sich dabei an aktuellen Empfehlungen der American Academy of Breastfeeding Medicine (ABM), die auch die späten Frühgeborenen mit einbeziehen. Diese Patientengruppe rückte in den letzten Jahren nicht nur zunehmend in den wissenschaftlichen Fokus, sondern ist auch im Beschluss des gemeinsamen Bundesausschusses zur Qualitätssicherung in der Versorgung von Früh- und Neugeborenen enthalten. Späten Frühgeborenen steht damit eine 24-stündige pädiatrische Facharztbetreuung zu, auch wenn sie, wie es im Konsensuspapier heißt: oft in der Wochenbettstation mitbetreut werden. Die Empfehlungen aus Österreich unterstützen die Kriterien der „baby-friendly hospital initiative“, der sich auch in Deutschland zunehmend Kliniken anschließen, und sollten damit eine breite Basis im Team aus Pädiatern, Pflegepersonal und Hebammen finden. Wenn zugefüttert werden muss, sollte dies, wenn abgepumpte Muttermilch nicht zur Ver- fügung steht, möglichst mit Frauenmilch (und erst an dritter Stelle mit Formulanahrung) erfolgen. Vor allem die neuen Bundesländer waren und sind in dieser Hinsicht aktiv. Dieses zweite Konsensuspapier entspricht in seiner praktischen und detaillierten Art dem Ersten. Dass die Ernährung Früh- und Neugeborener zu wichtig ist, um sie nur einer Berufsgruppe zu überlassen, wird im abschließenden Satz des Konsensuspapiers betont: Bei einigen wenigen medizinischen Indikationen ist Zufüttern unbedingt nötig - tritt dieser Fall ein, sollte das betreuende Team nach einheitlichem standardisiertem Vorgehen handeln und das Zufüttern gut dokumentieren.

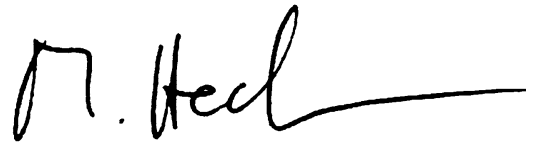

Prof. Dr. Matthias Heckmann

\section{Korrespondenzadresse}

Prof. Dr. M. Heckmann

Abteilung für Neonatologie und Pädiatrische Intensivmedizin, Zentrum für Kinder- und Jugendmedizin, Universitätsmedizin Greifswald Ferdinand-Sauerbruchstraße, 17475 Greifswald matthias.heckmann@uni-greifswald.de

Dieser Kommentar bezieht sich auf folgende Beiträge: - Ernährungskommission der Österreichischen Gesellschaft für Kinder- und Jugendheilkunde (ÖGKJ) (2012) Ernährung von Frühgeborenen nach der Entlassung. Monatsschr Kinderheilkd 160. DOI 10.1007/s0011201126189

- Ernährungskommission der Österreichischen Gesellschaft für Kinder- und Jugendheilkunde (ÖGKJ) (2012) Zufüttern bei gestillten reifen Neugeborenen und späten Frühgeborenen. Monatsschr Kinderheilkd 160. DOI 10.1007/ s0011201226433
Beikost umfassen. Seit kurzem stehen dafür auch in Deutschland spezielle Post- 
Hier steht eine Anzeige.

并 Springer 